\title{
Norepinephrine promotes glioma cell migration through up-regulating the expression of Twist1
}

\author{
Xue Wang ${ }^{1 *}{ }^{*}$, Ying Wang ${ }^{1+}$, Fang Xie ${ }^{1 \dagger}$, Zi-Tian Song ${ }^{2+}$, Zi-Qian Zhang ${ }^{2,3}$, Yun Zhao ${ }^{1}$, Shi-Da Wang ${ }^{1}$, Hui Hu ${ }^{1}$, \\ Yan-Shu Zhang ${ }^{2}$ and Ling-Jia Qian ${ }^{1^{*}}$
}

\begin{abstract}
Background: Glioma cells are characterized by high migration ability, resulting in aggressive growth of the tumors and poor prognosis of patients. It has been reported that the stress-induced hormone norepinephrine (NE) contributes to tumor progression through mediating a number of important biological processes in various cancers. However, the role of NE in the regulation of glioma migration is still unclear. Epithelial-to-mesenchymal transition (EMT) is one of the most important steps for tumor migration and metastasis. Twist1, as a key regulator of EMT, has been found to be elevated during glioma migration. But it is still unknown whether Twist1 is involved in the effect of NE on the migration of glioma cells.
\end{abstract}

Methods: Wound healing assay and transwell assay were conducted to evaluate the migration of glioma cells upon different treatments. The mesenchymal-like phenotype and the expression of Twist1 after NE treatment were assessed by cell diameters, real-time PCR, western blot and immunofluorescence staining. The gain-and loss-of-function experiments were carried out to investigate the biological function of Twist1 in the migration induced by NE. Finally, the clinical significance of Twist1 was explored among three public glioma datasets.

Results: In this study, our finding revealed a facilitative effect of NE on glioma cell migration in a $\beta$-adrenergic receptor (ADRB)-dependent way. Mechanistically, NE induced mesenchymal-like phenotype and the expression of Twist1. Twist1 overexpression promoted glioma cells migration, while knockdown of Twist1 abolished the discrepancy in the migration ability between NE treated glioma cells and control cells. In addition, the clinical analysis demonstrated that Twist1 was up-regulated in malignant gliomas and recurrent gliomas, and predicted a poor prognosis of glioma patients.

Conclusions: NE enhanced the migration ability of glioma cells through elevating the expression of Twist1. Our finding may provide potential therapeutic target for protecting patients with glioma from the detrimental effects of stress biology on the tumor progression.

Keywords: Norepinephrine, Glioma, Cell migration, Twist1

*Correspondence: snowwang0326@foxmail.com; newiia@vip.sina.com ${ }^{\dagger}$ Xue Wang, Ying Wang, Fang Xie and Zi-Tian Song contributed equally to this work.

${ }^{1}$ Institute of Military Cognition and Brain Sciences, Academy of Military Medical Sciences, \#27 Taiping Road, Haidian, Beijing 100850, China Full list of author information is available at the end of the article

\section{Background}

Malignant glioma is the most common primary intracranial tumor, accounting for approximately $80 \%$ of central nervous system (CNS) malignancies [1]. At present, the treatment of glioma is still mainly based on surgery to remove the tumor, and supplemented by radiation 
therapy, chemotherapy and other comprehensive treatment methods [2]. However, despite these treatments, the median survival of the patients with glioblastoma multiforme (GBM), which is the most malignant glioma [3], is approximately only 1 to 2 years [4]. Strong migration ability is one of the most important characteristics of glioma cells, resulting in aggressive growth and metastasis of the tumors, thus it is difficult to be completely removed by surgery $[5,6]$. Therefore, it is urgent to deeply investigate the regulatory mechanism of glioma migration and explore new therapeutic targets.

Patients with cancer suffer from persistent strong mental and physical stress, which causes adverse stress reactions of the body and affects clinical treatment and prognosis seriously. A large number of epidemiological investigations and experimental studies have shown that chronic stress is closely related to the occurrence and development of a variety of tumors [7-10]. Chronic stress can induce aberrantly persistent activation of the Sympathetic Nervous System (SNS), leading to the simultaneous elevation of catecholamines [11]. It has been reported that the stress-induced hormones, especially norepinephrine (NE), contribute to tumor progression through mediating a number of important biological processes, such as inducing anti-apoptosis activity and chemotherapy resistance, disrupting antitumor immunity and promoting angiogenesis [12-15]. In addition, propranolol, a $\beta$-adrenergic receptor (ADRB) antagonist, has been demonstrated to inhibit tumor growth and metastasis, which provides a new strategy for the treatment of tumors $[16,17]$. However, the reports about the involvement and the regulatory mechanism of $\mathrm{NE}$ in glioma are still rare.

In the present study, we explored the role of NE in the regulation of glioma cell migration, and elucidated that NE promoted glioma cell migration through binding to ADRBs. Mechanistically, NE induced mesenchymal-like phenotype and Twist1 expression. Twist1 overexpression facilitated the glioma cell migration, while knockdown of Twist1 abrogated the effect of NE on glioma cell migration. Moreover, our data revealed that Twist1 was upregulated in malignant gliomas and recurrent gliomas, and predicted a poor prognosis of glioma patients.

\section{Methods}

\section{Cell culture and intervention}

The glioma cell lines U251 and LN229 were purchased from Chinese National Infrastructure of Cell Line Resource. The two cell lines were authenticated by STR analysis. The cells were cultured in DMEM medium (Sigma, St. Louis MO, USA) containing 10\% FBS (Gibco, NY, USA), $100 \mathrm{U} / \mathrm{ml}$ penicillin and $100 \mathrm{U} / \mathrm{ml}$ streptomycin (HyClone, L.A., USA). All cells were grown in humidified incubator with $5 \% \mathrm{CO}_{2}$ at $37^{\circ} \mathrm{C}$.

Twist 1 expressing lentiviral vector was constructed by inserting the coding sequence (CDS) of human Twist1 gene (NM000474) in lentiviral GV358 vector (Genechem, Shanghai, China) which contained $3 \times$ flag and green fluorescent protein (GFP). The shRNA oligonucleotides (oligos) targeting Twist1 were synthesized by Genechem Co.,Ltd. (Shanghai, China). The sequences of the synthesized oligos were as follows: 5'-CCGGTCCGCAGTCTT ACGAGGAGCTCTCGAGAGCTCCTCGTAAGACTG CGGATTTTTG-3' (forward) and 5'-AATTCAAAAATC CGCAGTCTTACGAGGAGCTCTCGAGAGCTCCTC GTAAGACTGCGGA-3' (reverse). After annealing, double-strand oligos were inserted to lentiviral GV248 vector (Genechem, Shanghai, China). To produce lentivirus, HEK-293 T cells were co-transfected with the lentiviral vector described above and packaging vectors psPAX2 and VSVG using lipo2000 (Invitrogen, California, USA). The shRNA control virus and overexpression control virus were purchased from Genechem Co.,Ltd. (Shanghai, China).

Stable transfectants of glioma cells expressing Twist1 or a shRNA against Twist1 were established as described previously [18]. Briefly, U251 and LN229 cells were dissociated with $0.5 \%$ trypsin and seeded into six-well plates, following by infected with Twist1 overexpression virus, Twist1 knockdown virus and their control virus, separately. Then the stable infectants were screened by puromycin for 2 weeks.

NE (Sigma, St. Louis MO, USA), ADRB agonist (Isoprenaline) (Selleck Chemicals, Houston, USA) and antagonist (Propranolol) (Sigma, St. Louis MO, USA) were dissolved in Dimethyl Sulfoxide (DMSO) (Sangon Biotech, Shanghai, China) at the concentration of $20 \mathrm{mmol} / \mathrm{L}$. Then, $0.5 \mu \mathrm{l} / \mathrm{mL}$ of the regents were added into the culture medium for intervention. Especially, propranolol was added $1 \mathrm{~h}$ before NE. The final concentration of all drugs was chosen based on successful activation/inhibition in previous publications: NE $(10 \mu \mathrm{mol} / \mathrm{L})$, isoprenaline $(10 \mu \mathrm{mol} / \mathrm{L})$, propranolol $(10 \mu \mathrm{mol} / \mathrm{L})[19$, 20]. An equal volume of DMSO was used as control. In detail, when the cells were treated with only one regent (NE, isoprenaline or propranolol alone), $0.5 \mu \mathrm{l} / \mathrm{mL}$ of DMSO was used as control. When the cells were treated with two regents (NE combined with isoprenaline or propranolol), $1 \mu \mathrm{l} / \mathrm{mL}$ of DMSO was used as control.

\section{Wound healing assay}

The procedure of wound healing assay was based on previous study [21]. The glioma cells were seeded in 6-well plates and cultured until they reached confluence. A wound was then created by manually scraping the cell 
monolayer with a $200 \mu \mathrm{L}$ pipette tip. The cultures were rinsed several times with medium to remove dislodged cells, and $2 \mathrm{~mL}$ of DMEM medium with $1 \%$ FBS containing compounds were added into each well. Cells that had migrated into the wound area were photographed at $0 \mathrm{~h}$ and $24 \mathrm{~h}$. After exporting images, the boundaries of the wound were indicated by drawing two straight lines, and the distance between the two lines was measured using Image $J$ as the wound width at $0 \mathrm{~h}$ (X0) and $24 \mathrm{~h}$ (X24). The travelled distance percentage was determined as $(\mathrm{X} 0-\mathrm{X} 24) / \mathrm{X} 0 \times 100 \%$.

\section{Transwell assay}

To investigate cell migration ability, transwell assay was conducted using a 24-well insert with $8 \mu \mathrm{m}$ pores (Corning, NY, USA) as previously reported [22]. In total, $5 \times 10^{4}$ cells were seeded into the upper chamber of a polycarbonate transwell filters in $200 \mu \mathrm{L}$ serum-free DMEM medium. The lower chamber was added with DMEM medium containing 20\% FBS as chemoattractant. NE $(10 \mu \mathrm{mol} / \mathrm{L})$ or equal volume DMSO was added into the upper chamber. The cells were incubating for $6 \mathrm{~h}$. The chambers were fixed with $10 \%$ neutral formalin for $15 \mathrm{~min}$ following by removing the non-migratory cells with cotton swabs gently. The cells were then dyed with crystal violet (Beyotime, Shanghai, China). Images were acquired using a microscope (Olympus, Tokyo, Japan). Then the number of cells of each field was counted manually. Besides, the staining areas were measured using Image-Pro plus 6.0. Three independent experiments were conducted and the data was presented as the means \pm SD.

\section{Real time PCR}

Total RNA from glioma cells was extracted using TRIzol reagent (Invitrogen California, USA). Synthesis of cDNA was done using the M-MLV Reverse Transcriptase (Promega, Wisconsin, USA) according to the manufacturer's instructions. Real-time PCR was performed using a LightCycler 96 Realtime PCR System (Roche, Basel, Switzerland) with TB Green Premix Ex Taq kit (TaKaRa, Kyoto, Japan). The $\beta$-actin was selected as the endogenous control in the assay. Relative levels of indicated genes were calculated by $2^{-\Delta \Delta \mathrm{Ct}}$ method as reported previously [23]. The primer sequences are provided as following:

\begin{tabular}{lll}
\hline N-cadherin & Forward $\left(5^{\prime}-3^{\prime}\right)$ & AGGATCAACCCCATACACCA \\
& Reverse $\left(5^{\prime}-3^{\prime}\right)$ & TGGTTTGACCACGGTGACTA \\
Fibronectin1 & Forward $\left(5^{\prime}-3^{\prime}\right)$ & CAGTGGGAGACCTCGAGAAG \\
& Reverse $\left(5^{\prime}-3^{\prime}\right)$ & TCCCTCGGAACATCAGAAAC \\
\hline
\end{tabular}

\begin{tabular}{lll}
\hline Vimentin & Forward $\left(5^{\prime}-3^{\prime}\right)$ & GAGAACTTTGCCGTTGAAGC \\
& Reverse $\left(5^{\prime}-3^{\prime}\right)$ & TCCAGCAGCTTCCTGTAGGT \\
Twist1 & Forward $\left(5^{\prime}-3^{\prime}\right)$ & GGAGTCCGCAGTCTTACGAG \\
& Reverse $\left(5^{\prime}-3^{\prime}\right)$ & TCTGGAGGACCTGGTAGAGG \\
-actin & Forward $\left(5^{\prime}-3^{\prime}\right)$ & AATCGTGCGTGACATTAAGGAG \\
& Reverse $\left(5^{\prime}-3^{\prime}\right)$ & ACTGTGTTGGCGTACAGGTCTT \\
\hline
\end{tabular}

\section{Immunofluorescence staining}

Glioma cells were treated with NE or DMSO for $24 \mathrm{~h}$ and then washed with TBS for 2 times. Immunofluorescence staining was conducted based on previously reported [24]. The cells were fixed in $4 \%$ paraformaldehyde for $15 \mathrm{~min}$, and permeabilized with $0.4 \%$ Triton X-100 for $5 \mathrm{~min}$. After washing with TBS for 3 times, the cells were blocked with PBS containing $10 \%$ goat FBS (Solarbio, Beijing, China) and 1\% BSA for $1 \mathrm{~h}$ and subsequently incubated with rabbit anti-human Twist1 antibody (1:50, Proteintech, Chicago, USA) at $4{ }^{\circ} \mathrm{C}$ overnight. After washed with TBS, the cells were incubated with Rhodamine-conjugated goat anti-rabbit IgG antibody (1:100, Proteintech, Chicago, USA) at room temperature for $30 \mathrm{~min}$ and then counterstained with $4^{\prime}, 6$-diamidino2-phenylindole (DAPI) (Solarbio, BeiJing, China). Images were captured using a fluorescence microscope (Olympus, Tokyo, Japan).

\section{Western blot analysis}

Western blot analysis was performed as described previously [25]. The cells were lysed with Radio Immunoprecipitation Assay (RIPA) cell lysate (Solarbio, Beijing, China) containing protease inhibitors, and centrifuged at $12,000 \mathrm{~g}$ for $15 \mathrm{~min}$ at $4^{\circ} \mathrm{C}$ to extract the supernatant. Then loading buffer (Tiangen, Beijing, China) was added and boiled for $5 \mathrm{~min}$ to denaturation. The samples were electrophoretic in 10\% SDS-PAGE gel, following by transferred to poly vinylidene fluoride (PVDF) membrane using wet transfer system (Bio-rad, California, USA). The membrane was sealed in $5 \%$ milk at room temperature for $1 \mathrm{~h}$ and washed 3 times in $1 \times$ TBST. The membrane was cut prior to hybridisation with antibodies. The primary antibodies were added separately and incubated overnight at $4^{\circ} \mathrm{C}$. After washing 3 times in $1 \times$ TBST, the HRP labeled secondary antibodies were incubated for $2 \mathrm{~h}$ at room temperature. The membranes were prepared with ECL Western blotting substrate Kit (Thermo Scientific, Massachusetts, USA) followed with image acquisition by Image Quantlas 4000 (GE, NY, USA) and analysis by Image Quantlas 4000 and Image J. The original images of the blots shown in the figures could be found in the Supplementary Information file. The antibodies used in the study were Rabbit anti-Fibronection (1:500, Proteintech, Chicago, USA), N-cadherin (1:1000, Proteintech, 
Chicago, USA), Vimentin (1:3000, Proteintech, Chicago, USA), mouse anti $\beta$-actin (1:5000, Proteintech, Chicago, USA), Goat anti rabbit IgG (1:5000, ZSGB-Bio, Beijing, China) and Goat anti mouse IgG (1:5000, ZSGB-Bio, Beijing, China).

\section{Patient datasets and clinical analysis}

The Glioma French dataset [26] and the cancer genome atlas (TCGA) dataset used in this study were publicly available from the R2: Genomics Analysis and Visualization Platform (http://r2.amc.nl). The expression of Twist1 in the two datasets and the survival analysis of patients from the TCGA dataset were also analyzed using this platform. In addition, the Chinese Glioma Genome Atlas (CGGA) dataset (mRNAseq_693) [27, 28] used in this study was collected and analyzed at CGGA (http://www. cgga.org.cn/).

\section{Data analysis}

Statistical analysis was processed with SPSS 23.0 software. The data was expressed as mean \pm SD. The continuous variables were evaluated for normality before comparison for statistical differences. Student's t-test was used for comparison between two groups. One-way ANOVA analysis was performed to compare the values among multiple groups. Signifcant results of ANOVA were subjected to Bonferroni post hoc test. All differences were two-sided. A $p$-value less than 0.05 was considered to have significant difference.

\section{Results}

\section{NE promoted glioma cells migration} in an ADRB-dependent way

The U251 and LN229 cells were treated with NE to investigate the effect of stress hormone on glioma cell behavior in vitro. Wound healing assay was used to examine the cell migration ability. Compared with the control group, the rates of travelled distance in the cells after NE treatment for $24 \mathrm{~h}$ were elevated (Fig. 1A, Student's t-test, U251, $p=0.043$; LN229, $p=0.008$ ). Consistently, the results of transwell assay also showed that both the number of migrated cells (U251, $p=0.002$; LN229, $p=0.041$ ) and the staining area (U251, $\mathrm{p}=0.002 ; \mathrm{LN} 229, p=0.033)$ were increased upon NE treatment (Fig. 1B \& Supplementary Fig. 1A, Student's t-test). Studies have found that NE primarily binds to ADRB to exert its biological effect in various cancers such as ovarian, prostate and pancreatic cancer [10, 12, 29]. Isoprenaline, an ADRB agonist, was further utilized to evaluate the role of ADRB in glioma cells migration. The data of wound healing assay revealed that isoprenaline elevated the migration ability of U251 ( $p=0.002)$ and LN229 ( $p=0.006)$ (Fig. 1C, Student's t-test). Besides, isoprenaline combined with NE could also promote the migration of glioma cells (Supplementary Fig. 1B, Student's t-test, U251, $p=0.004$; LN229, $p=0.007)$. Furthermore, the ratio of isoprenaline+NE/ DMSO was increased compared with that of NE/DMSO or isoprenaline/DMSO in the travelled distance, suggesting a stronger role of isoprenaline combined with $\mathrm{NE}$ in the comparison with NE or isoprenaline alone in the migration of glioma cells (Supplymentary Fig. 1C, Oneway ANOVA, U251, $p=0.0001$; LN229, $p=0.003$ ). In addition, an ADRB antagonist propranolol was demonstrated to restrain the migration triggered by NE (Fig. 1D, One-way ANOVA, U251, $p=0.002$; LN229, $p=0.001$ ), although the propranolol alone showed no obvious effect on the cells migration (Supplementary Fig. 1D, Student's t-test, U251, $p=0.449$; LN229, $p=0.892$ ). Thus, our findings indicated that the stress hormone NE promoted glioma cells migration in an ADRB-dependent way.

\section{NE induced mesenchymal-like phenotype of glioma cells}

The epithelial-to-mesenchymal transition (EMT) is a critical event in mediating tumor migration, invasion and metastasis. Whether NE could regulate the EMT phenotype thus promote the migration of glioma cells is still unknown. EMT is characterized by cell morphologic changes from irregular polygons to elongated shape. So we determined the cell diameters after NE treatment. As shown in Fig. 2A \& B, the cell diameters were significantly increased upon NE treatment compared with the control group (Student's t-test, U251, $p=0.0001$; LN229, $\mathrm{p}=0.0001)$. Moreover, the results of real-time PCR assay showed that compared with DMSO group, the mRNA levels of mesenchymal markers including N-cadherin (U251, $p=0.027$; LN229, $p=0.003$ ), Fibronectin1 (U251, $p=0.031$; LN229, $p=0.006)$ and Vimentin (U251, $p=0.012$; LN229, $p=0.018$ ) were up-regulated after $\mathrm{NE}$ intervention (Fig. 2C, Student's t-test). Western blot results also revealed a various increase on the protein levels of N-cadherin, Fibronectin1 and Vimentin, which further confirmed a positive role of NE on the expression of mesenchymal markers (Fig. 2D \& Supplementary Fig. 2). The aforementioned results suggested an induction of the mesenchymal-like phenotype by NE in U251 and LN229 cells.

\section{NE up regulated the expression of Twist 1}

We further examined the influence of NE on Twist1 expression, which is one of the key regulators of EMT progress. As shown in Fig. 3A, the mRNA level of Twist1 was raised in NE-treated cells compared to the control cells (Student's t-test, U251, $p=0.024$; LN229, $p=0.002$ ). Consistently, NE treatment also increased the expression of Twist1 protein (Fig. 3B \& Supplementary Fig. 3A\&B, Student's t-test, U251, $p=0.007$; LN229, $p=0.005$ ). 


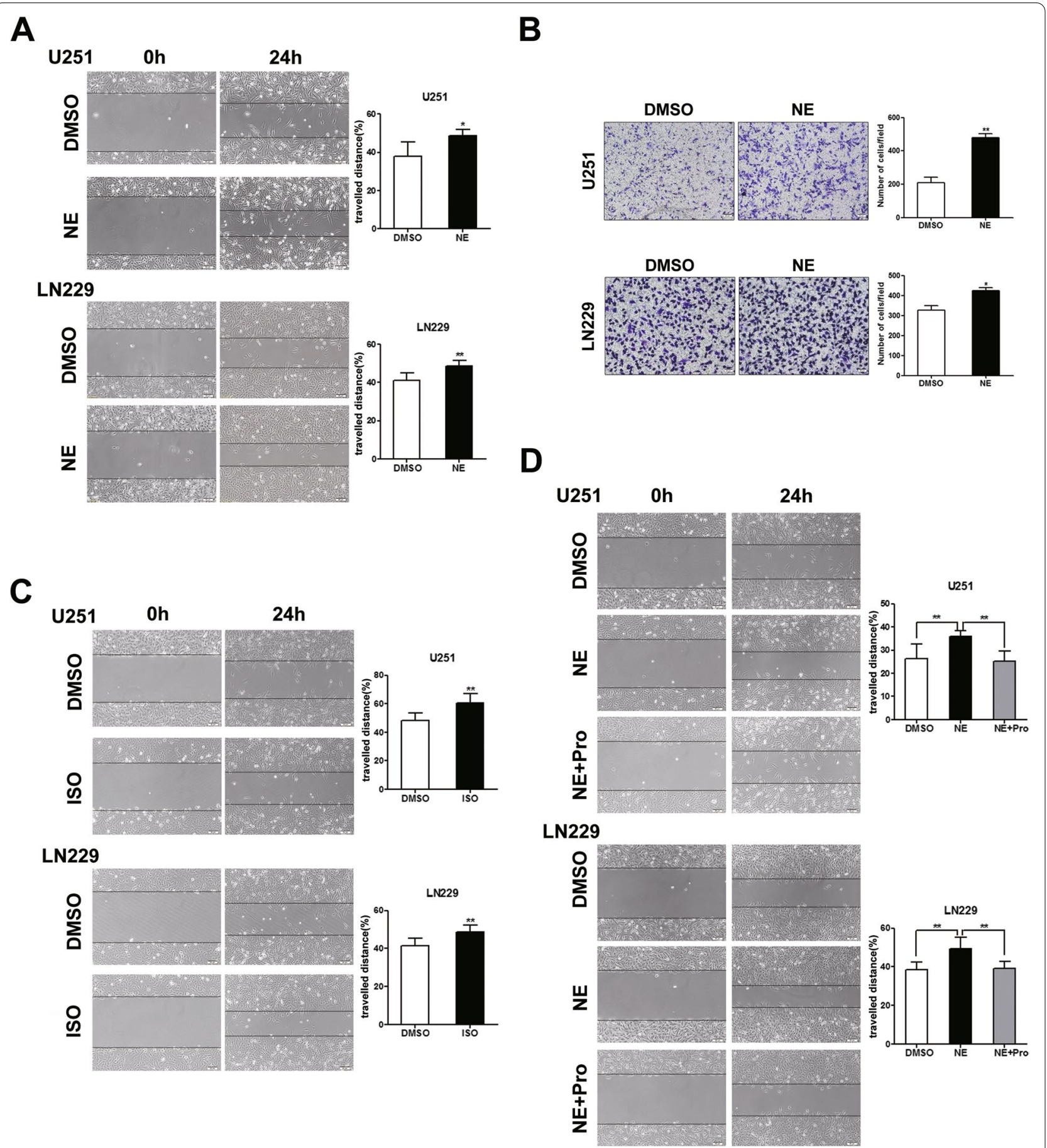

Fig. 1 NE promoted glioma cells migration via ADRB. Wound healing assays and transwell assays were used to evaluate the effects of NE administration on glioma cells migration. A Representative images and the higher travelled distances of wound healing in NE-treated U251 ( $n=4$, $p=0.043)$ and LN229 $(n=4, p=0.008)$ cells. Scale bar, $50 \mu$ m. Student's t-test was used for statistical analysis. B Representative images and the increased cell number of transwell assays in NE-treated U251 $(n=3, p=0.002)$ and LN229 $(n=3, p=0.041)$ cells. Scale bar, $50 \mu m$. Student's t-test was used for statistical analysis. C Representative images and isoprenaline (ISO, $10 \mu \mathrm{mol} / \mathrm{L})$ elevated the migration ability of U251 ( $n=6, p=0.002)$ and LN229 ( $n=6, p=0.006)$. Scale bar, $50 \mu \mathrm{m}$. Student's t-test was used for statistical analysis. D Representative images and propranolol (Pro, $10 \mu \mathrm{mol} / \mathrm{L})$ administration reversed the effect of NE on migration of $\mathrm{U} 251(n=6, p=0.002)$ and LN229 $(n=6, p=0.001)$. Scale bar, $50 \mu \mathrm{m}$. One-way ANOVA analysis was used for statistical analysis. ${ }^{*} p<0.05,{ }^{* *} p<0.01$ 


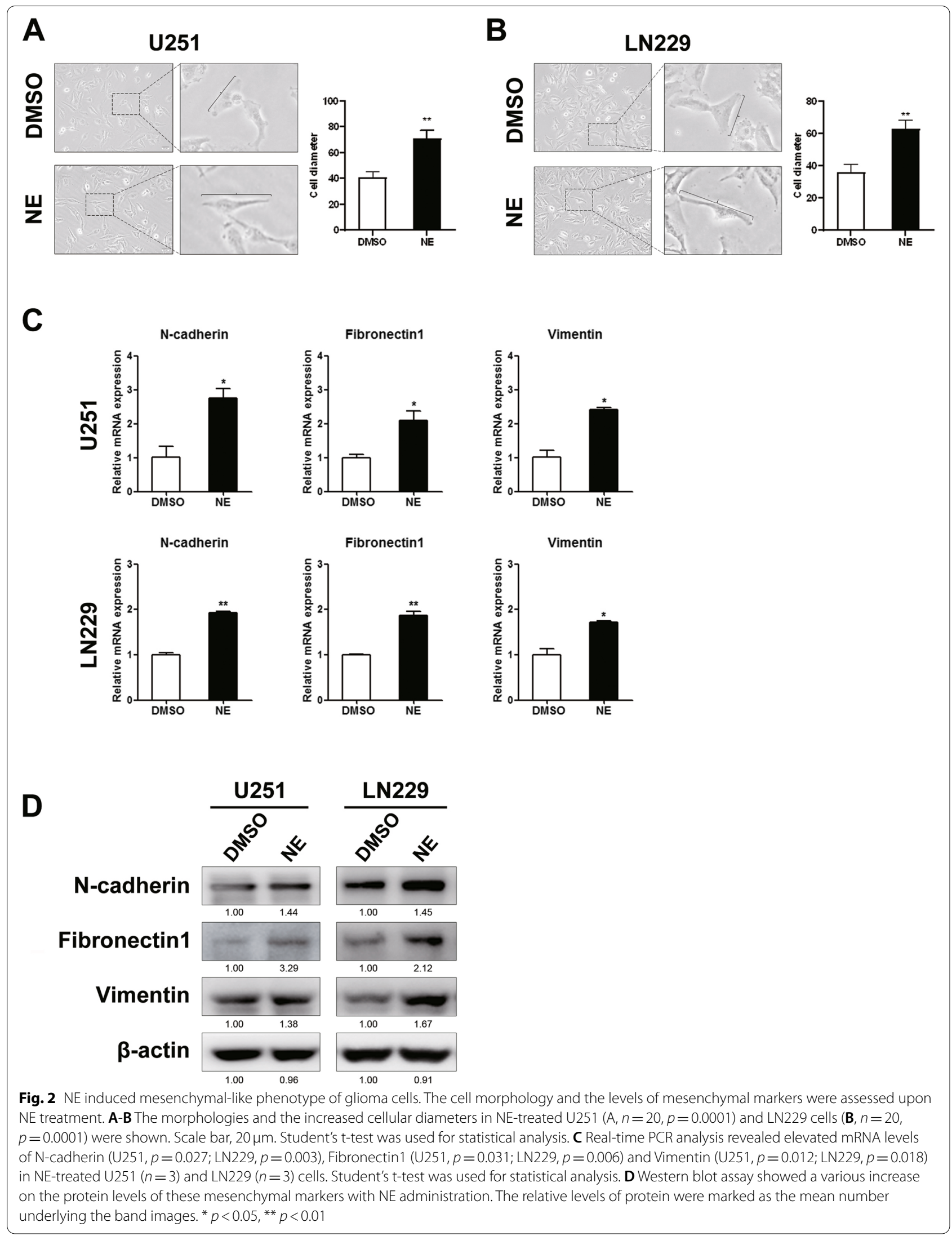




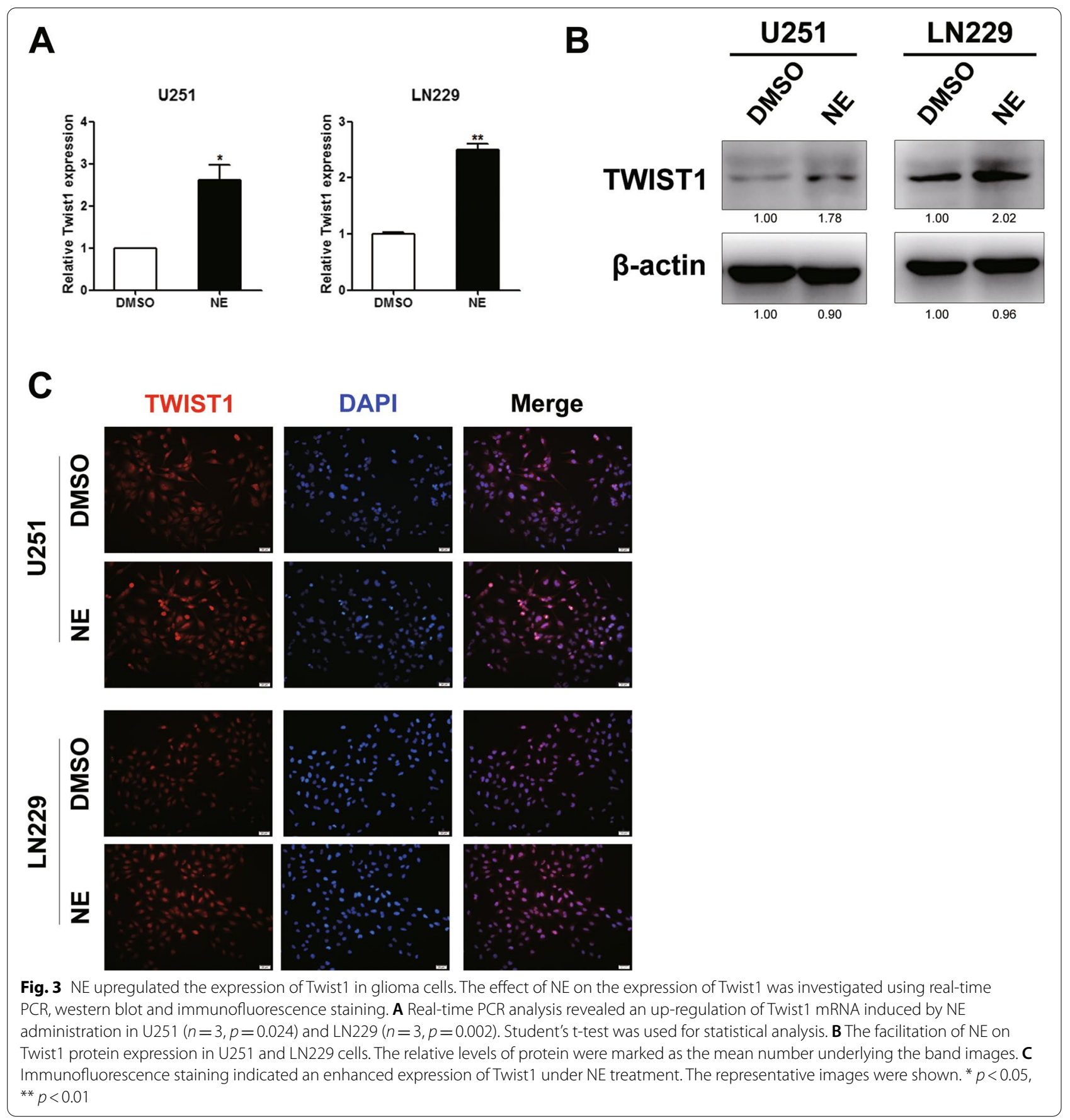

What's more, the enhanced expression of Twist 1 in the $\mathrm{NE}$ group was further validated by immunofluorescence staining (Fig. 3C).

Twist 1 facilitated mesenchymal-like phenotype and migration of glioma cells

Next, the U251 and LN229 cells were infected with Twist1 overexpression virus and control virus. The overexpression effect was confirmed by real-time PCR and western blot assay (Fig. 4A\&B, Student's t-test, U251, $p=0.038$; LN229, $p=0.005$ ). The role of Twist1 in regulating the migration of glioma cells was further explored. Compared with the control cells, Twist1 overexpression enhanced the mRNA levels of mesenchymal markers including $\mathrm{N}$-cadherin (U251, $p=0.016$; LN229, $p=0.011$ ), Fibronectin1 (U251, $p=0.014 ; \mathrm{LN} 229$, 
$p=0.037)$ and Vimentin (U251, $p=0.021 ;$ LN229, $p=0.008$ ) (Fig. 4C, Student's t-test). The results of western blot assay showed a various elevation on the protein levels of mesenchymal markers under Twist1 overexpression. Specifically, compared with the control cells, N-cadherin and Fibronectin1 increased significantly while Vimentin increased slightly in U251-LVTwist1 cells. And the protein levels of mesenchymal markers were promoted mildly in LN229-LVTwist1 cells (Fig. 4D). Moreover, the results of wound healing assay also revealed that Twist 1 overexpression facilitated the migration of glioma cells (Fig. 4E\&F, Student's t-test, U251, $p=0.018$; LN229, $p=0.002$ ).

\section{Twist 1 depletion restrained NE-induced glioma cells migration}

To further characterize the role of Twist1 in NE-mediated glioma cells migration, stable transfectants of glioma cells expressing shRNA against Twist1 were established. The knockdown effects were determined by real-time PCR and western blot assay (Fig. 5A\&B \& Supplementary Fig. 5, Student's t-test, U251, $p=0.036$; LN229, $p=0.005)$. As expected, knockdown of Twist1 alleviated the distinct expression levels of mesenchymal makers between glioma cells upon NE treatment and DMSO treatment (Fig. 5C\&D, One-way ANOVA, U251, $\mathrm{N}$-cadherin $p=0.014$, Fibronectin $p=0.016$, Vimentin $p=0.021 ; \mathrm{LN} 229$, N-cadherin $p=0.037$, Fibronectin $p=0.011$, Vimentin $p=0.008)$. Consistently, Twist1 depletion also abrogated the discrepancy of migration between the NE treated glioma cells and the control cells (Fig. 5E\&F, One-way ANOVA, U251, $p=0.0001$; LN229, $p=0.001)$. Taken together, these results supported that knockdown of Twist1 inhibited glioma cells migration under NE treatment, indicating a regulative role of Twist1 in NE-mediated glioma cells migration.

\section{Twist 1 was up-regulated in human gliomas and predicted a poor prognosis of glioma patients}

To explore the clinical significance of Twist1, we analyzed the levels of Twist1 in glioma tissues and normal tissues, different World Health Organization (WHO) tumor grades and different subtypes using multiple datasets. The result showed that the expression of Twist1 was higher in glioma samples than in control samples (Fig. 6A, Student's t-test, $p=0.014$ ). Besides, the expression of Twist1 was significantly increased from the WHO tumor grade II to grade IV, suggesting a correlation between the level of Twist1 and the malignant degree of glioma (Fig. 6B, One-way ANOVA, $p=1.22 \mathrm{e}-14$ ). The analysis of the TCGA data set indicated that Twist1 was hyper-expressed in mesenchymal subtypes compared to proneural, neural and classical subtypes of glioma tumors (Fig. 6C, One-way ANOVA, $p=4.62 \mathrm{e}-04$ ). As tumor migration has been found to be closely related to tumor relapse, we further analyzed the level of Twist 1 in primary and recurrent gliomas. The result showed that Twist1 expression was up-regulated in the recurrent gliomas compared to the primary gliomas (Fig. 6D, Student's $\mathrm{t}$-test, $p=7.12 \mathrm{e}-09$ ). In addition, the overall survival (OS) analysis demonstrated that Twist1 high expression in gliomas predicted short survival of patients by analyzing the TCGA database (Fig. 6E left \& Supplementary Fig. 6, Kaplan-Meier method, $p=0.02 \& 0.026$, respectively) and CGGA datebase (Fig. 6E right, Kaplan-Meier method, $p=7.12 \mathrm{e}-09$ ).

\section{Discussion}

Malignant gliomas are highly infiltrative tumors, with cells migrating from the primary lesion into surrounding normal brain tissue, leading to complicating complete surgical resection, tumor recurrence and poor prognosis of patients. Thus, clarifying the underlying regulatory mechanism of glioma migration to identify novel targets for effective intervention is urgently needed [4]. In this study, our results showed that NE promoted glioma cells migration and induced mesenchymal-like phenotype. Furthermore, the biological effect of NE on glioma cells migration was attributed to the binding with ADRB and the regulation of Twist1 expression.

Epidemiological and experimental studies have shown that stress can modulate the progression of tumor, particularly, one large meta-analysis on 163,363 individuals published in 2017 revealed that self-reported

\footnotetext{
(See figure on next page.)

Fig. 4 Twist1 overexpression facilitated mesenchymal-like phenotype and migration of glioma cells. Lentiviruses were used to establish the stable glioma cell lines with Twist1 overexpression. The levels of Twist1, mesenchymal markers and the migration ability were measured in the indicated cells. A The overexpression of Twist1 in established U251 cell lines were validated by real-time PCR (left panel, $n=3, p=0.038$ ) and western blot (right panel). Student's t-test was used for statistical analysis. B The overexpression of Twist1 in established LN229 cell lines were validated by real-time PCR (left panel, $n=3, p=0.005$ ) and western blot (right panel). Student's t-test was used for statistical analysis. $C$ Real-time PCR revealed the increased mRNA levels of the mesenchymal markers in U251/LN229 LVTwist1 cells (U251: $n=3$, N-cadherin $p=0.016$, Fibronectin1 $p=0.014$, Vimentin $p=0.021$; LN229: $n=3$, N-cadherin $p=0.011$, Fibronectin $1 p=0.037$, Vimentin $p=0.008$ ). Student's t-test was used for statistical analysis. D The higher expressed mesenchymal markers in LVTwist1 cells were shown. The relative levels of those markers were noted as the mean number underlying the band images. E Twist1 overexpression led to higher travelled distances of wound healing both in U251 $(n=5, p=0.018)$ and LN229 cells $(n=5, p=0.002)$. Scale bar $=50 \mu \mathrm{m}$. Student's t-test was used for statistical analysis. ${ }^{*} p<0.05,{ }^{* *} p<0.01$
} 
A

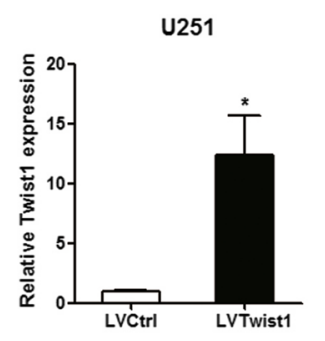

C
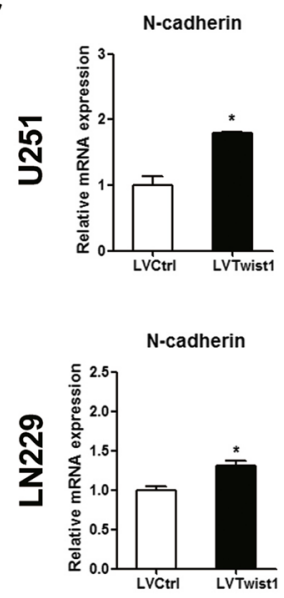

E
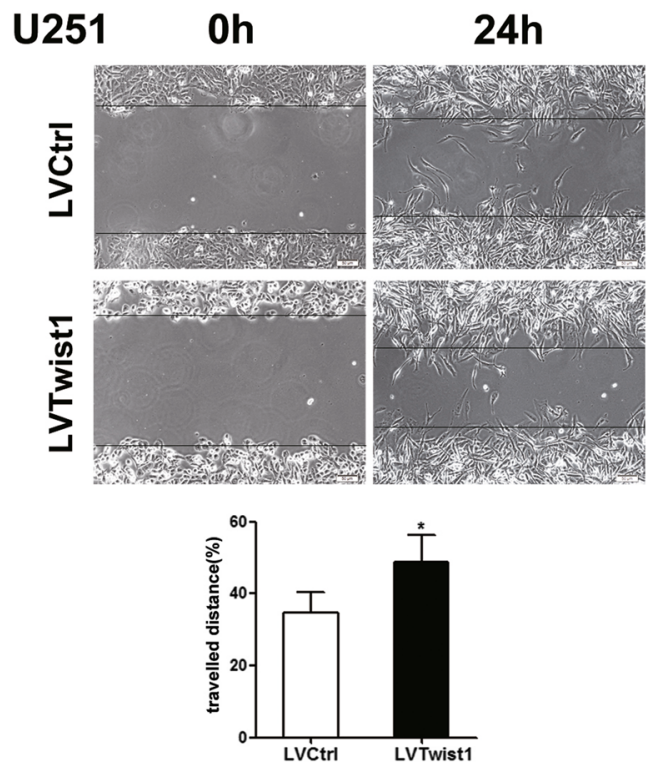

B
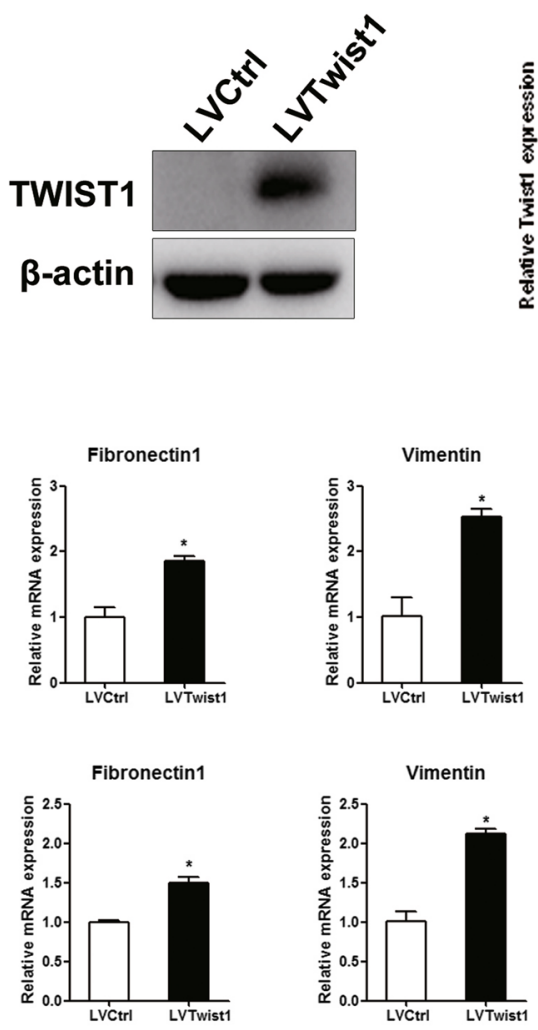
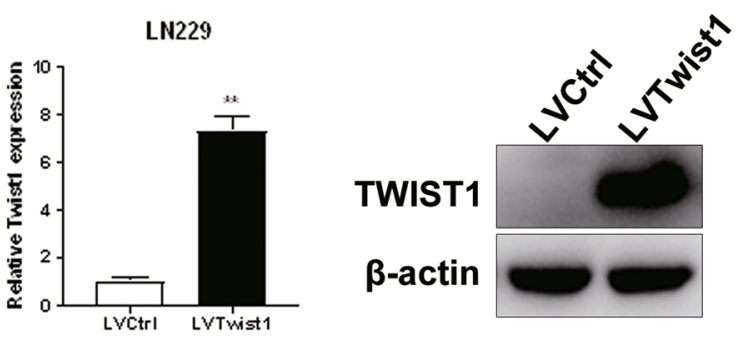

$\beta$-actin
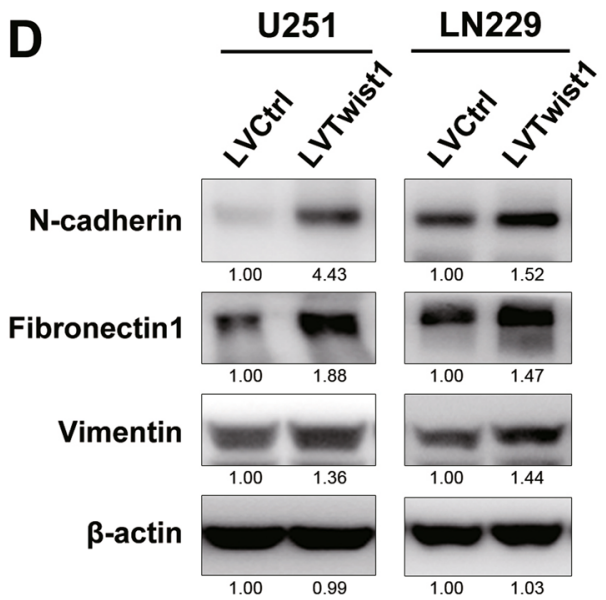

F
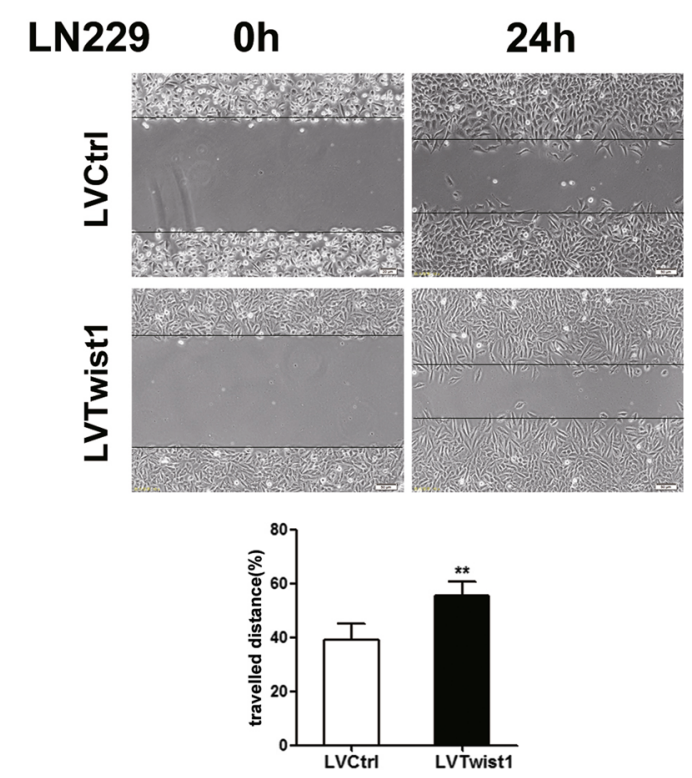

Fig. 4 (See legend on previous page.) 


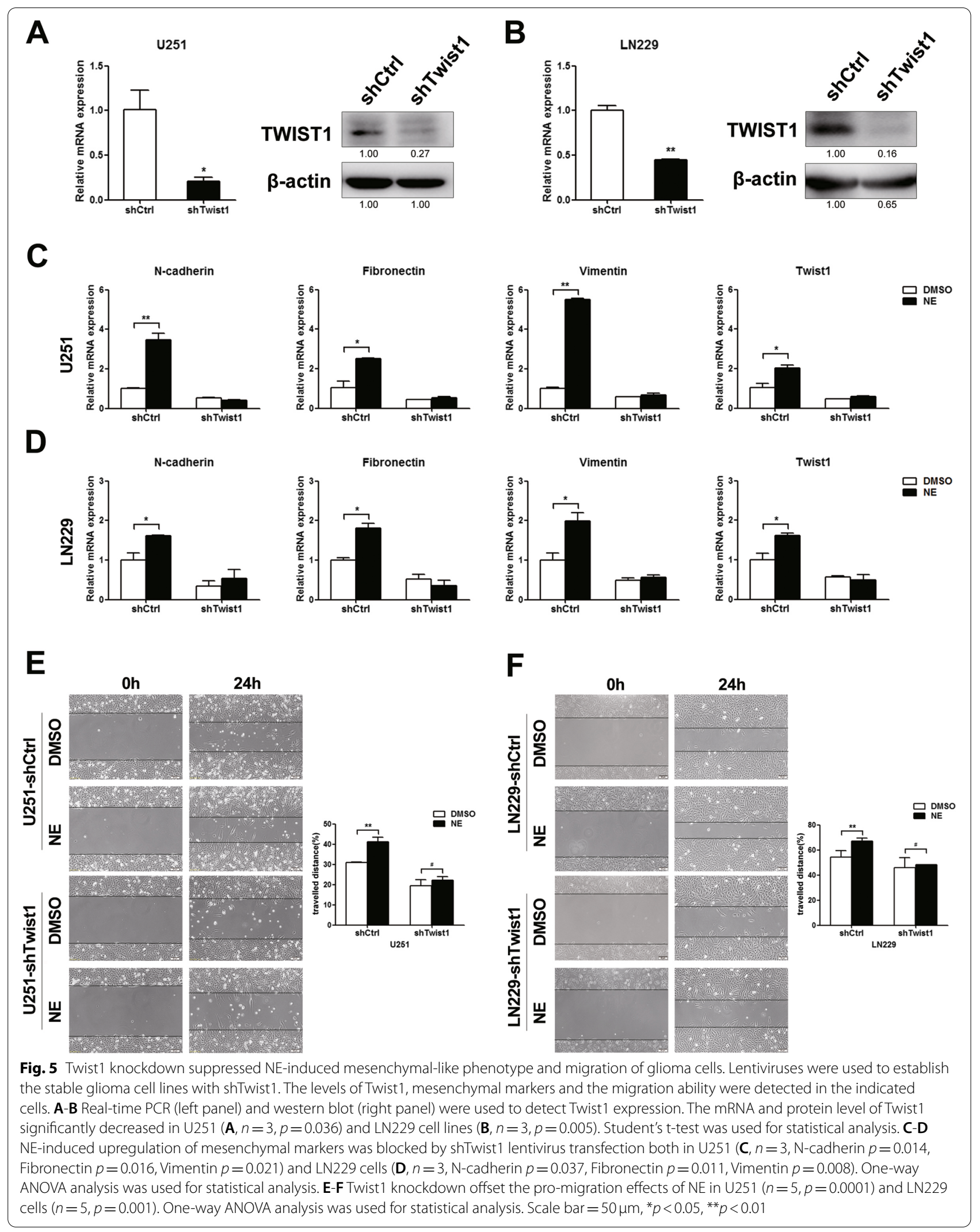


psychological-distress scores are epidemiologically linked to cancer-related mortality [30]. However, the biological mechanisms underlying such effects are not well understood. Data from animal models has shown that stress could affect immune responses via neuroendocrine system [14]. Nevertheless, recent studies indicated that stress mediators from the sympathetic nervous system could also have a direct effect on the malignant behavior of tumor cells independent of the dysregulation on the immune system [31]. The catecholamine hormone, $\mathrm{NE}$, is a crucial neurotransmitter released by the SNS in response to physiological, psychological, or environmental threats. It has been reported that NE could directly induce gene expression and was involved in invasion, migration, and metastasis in a variety of cancers [32-35]. However, little is known about the effect of NE on glioma cell migration. In this study, the data of wound healing assay showed that NE elevated the rate of travelled distance compared with the DMSO group. Consistently, the number of migrated cells was increased upon NE treatment evaluated by transwell assay. Our results suggested a promoting role of NE on glioma cells migration.

Families of adrenergic receptors mediate most of the biological effects of norepinephrine and can be divided into $\alpha$ - and $\beta$-adrenergic receptors. The growing evidence has suggested that ADRBs are predominantly responsible for the impact of NE and chronic stress on the progression of cancer [34]. Further, some studies have demonstrated that the expressions of ADRBs were higher in multiple types of tumor tissues compared with the corresponding normal tissues [36, 37]. Chronic stress and NE can accelerate tumor cell proliferation, angiogenesis and metastasis via the ADRB-mediated activation of cAMP/ PKA signaling and thus upregulating the level of VEGF, MMPs, the phosphorylation of Bad and many other signaling pathways $[10,35,38,39]$. So far, a growing number of studies have supported the use of $\beta$-blockers in reducing the rates of progression of several solid tumors and prolonging survival of cancer patients [40-43]. Propranolol, as an ADRB antagonist, has been demonstrated to inhibit tumor growth and metastasis, making it a new alternative for cancer adjuvant chemotherapy. In the present study, we found that ADRB agonist isoprenaline could increase the migration capacity of U251 and LN229 cells. In further, propranolol could significantly reduce the migration of glioma cells induced by NE. Our findings suggested that $\beta$-adrenergic blockade might play a role in glioma treatment, especially for the cancer patients suffering from the detrimental effects of chronic stress.

Tumor dissemination and metastatic behavior account for the vast majority of cancer associated mortality. Epithelial tumors achieve this progressive state via EMT, which has been shown to be an important step for tumor migration and metastasis [44]. During EMT, tumor cells gradually lose intercellular contacts and undergo morphological changes from epithelial cells to mesenchymal-appearing cells. The decreased epithelial marker E-cadherin and the up-regulated mesenchymal markers such as $\mathrm{N}$-cadherin, Vimentin, Fibronectin1 are believed to be hallmarks of EMT $[45,46]$. Studies have shown that glioma cells can undergo EMT or EMT(-like) changes to gain the ability of metastasis $[47,48]$. However, the majority of GBMs do not show intrinsic E-cadherin expression and the mesenchymal transition occurs independently from E-cadherin to $\mathrm{N}$-cadherin shift [49]. Similarly, we also found that the levels of E-cadherin were extremely low in the two glioma cell lines used in this research as the $\mathrm{Ct}$ values were more than 30 in realtime PCR analysis. Nevertheless, our data showed that compared with the control group, the cell diameters were increased after NE treatment, indicating a change in cell morphology. Furthermore, the expression of mesenchymal markers including $\mathrm{N}$-cadherin, Fibronectin1, Vimentin increased upon NE intervention, suggesting that NE could enforce the mesenchymal-like phenotype of glioma cells.

We also explored the molecular mechanism of NE regulated glioma cells migration. Members of the TWISTfamily are demonstrated to be crucial EMT-activators. While a great number of studies has implicated that Twist 1 is overexpressed in various carcinomas and plays a vital role in tumor initiation, stemness, angiogenesis,

(See figure on next page.)

Fig. 6 Expression and clinical correlation of Twist1 in glioma patients. The expression of Twist1 in clinical glioma samples and its relationship with patients'survival time were analyzed in different glioma datasets. A A high level of Twist 1 was observed in gliomas compared with control tissues from the French glioma database $(n=284, p=0.014)$. Student's t-test was used for statistical analysis. B The expression of Twist1 was increased from the WHO tumor grade II to grade IV in the CGGA glioma database $(n=692, p=1.22 \mathrm{e}-14)$. One-way ANOVA analysis was used for statistical analysis. C Twist1 expression was up-regulated in mesenchymal subtypes compared to other indicated subtypes of glioma tumors from the TCGA glioma database ( $n=170, p=4$.62e-04). One-way ANOVA analysis was used for statistical analysis. D Twist1 was hyper-expressed in the recurrent gliomas compared to the primary gliomas from the CGGA glioma database ( $n=693, p=7.12 \mathrm{e}-09$ ). Student's t-test was used for statistical analysis. E Kaplan-Meier survival analysis of patients from TCGA glioma database ( $n=504, p=0.02$, left panel) and CGGA glioma database $(n=668$, $p=7.12 \mathrm{e}-09$, right panel) stratified by Twist1 expression. A lower survival probability in high Twist1 expressed patients compared with those patients with low Twist1 expression was revealed. Median Twist1 expression was used for stratification into Twist1 high expression and Twist1 low expression tumors 
A

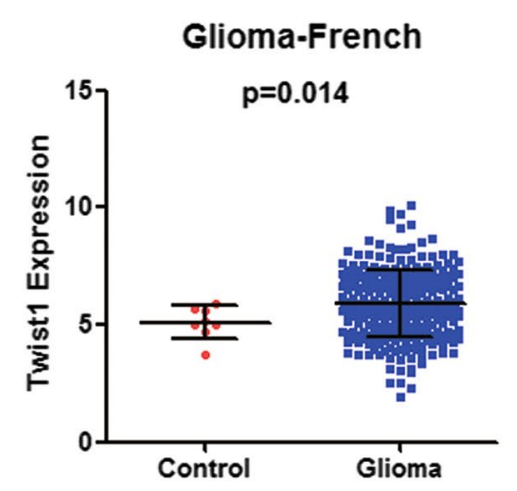

C

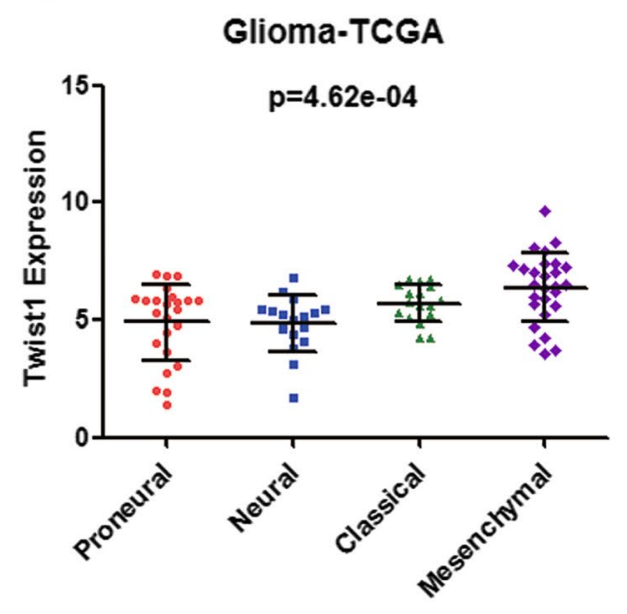

E
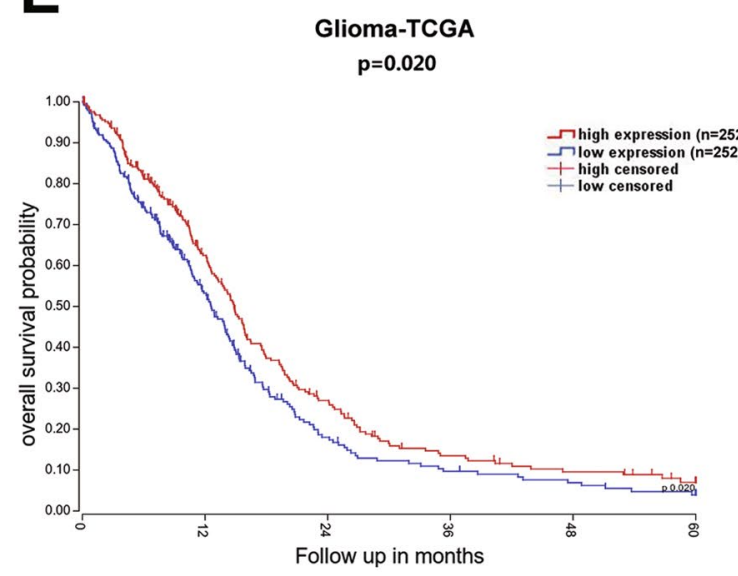

Fig. 6 (See legend on previous page.)
B

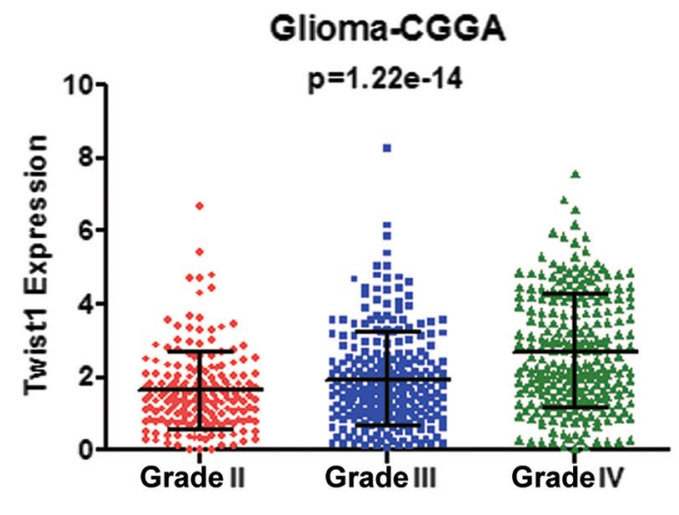

D
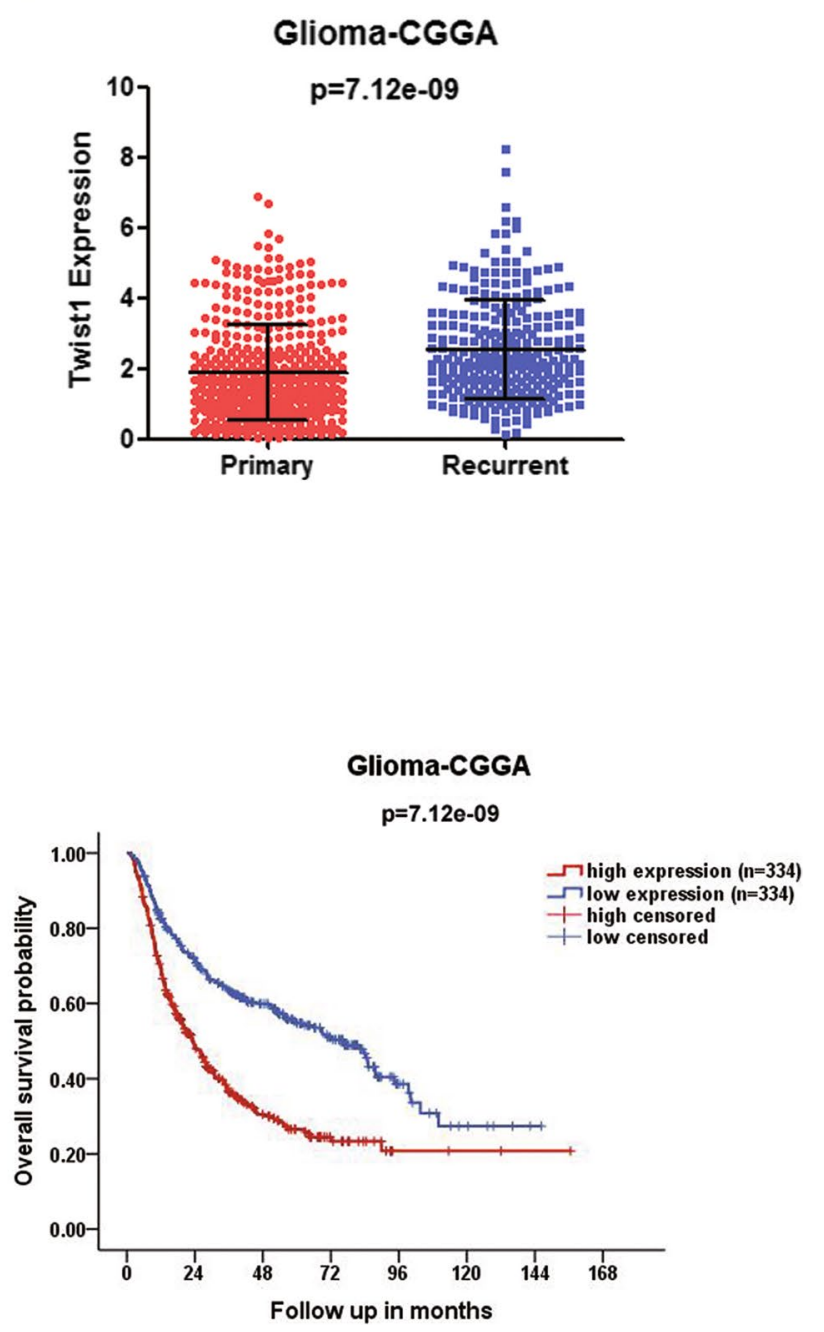
dissemination and chemoresistance [50]. More recently, emerging evidence has shown that elevated expression of Twist 1 was observed during the glioma cell invasion and migration [51]. Therefore, in this study we examined the influence of NE on Twist1 expression and found that NE increased the expression of Twist1 in U251 and LN229. We further used lentivirus to modulate the expression of Twist1. Consistent with previous reports, we also proved that overexpression of Twist1 could promote glioma cells migration. More importantly, our data revealed that knockdown of Twist1 abolished the migration trigged by NE. However, the molecular mechanism underlying high level of Twist1 under NE intervention remains unclear, which is one of the limitations of this study. Studies have shown that the expression of Twist1 is modulated by an array of different upstream regulators via multiple pathways including JAK2/STAT3, GSK-3 $\beta / \beta$-catenin, TGF- $\beta$ / Smad, PKB/AKT and NF-кB $[49,52-54]$. In another study of our team, we have found that PI3K/Akt signaling was activated upon $\mathrm{NE}$ administration in glioma cells [19]. As reported in other literatures, the expression of Twist1 is closely regulated by the PI3K/AKT signaling pathway in glioma and many other tumors [55-57]. These findings suggest that the PI3K/Akt pathway may play a regulate role in the NE-induced Twist1 promotion, which needs more investigation.

\section{Conclusions}

In summary, we have found that stress-induced hormones NE promoted glioma cell migration. Mechanistically, NE mainly bound to ADRB and further increased the expression of Twist1 to induced mesenchymal-like phenotype of glioma cells. Our findings may provide potential therapeutic targets and pave the way for the development of new strategies to protect patients with glioma from the detrimental effects of stress on tumor progression.

\begin{abstract}
Abbreviations
NE: Norepinephrine; EMT: Epithelial-to-mesenchymal transition; ADRB: $\beta$-adrenergic receptor; CNS: Central nervous system; GBM: Glioblastoma multiforme; SNS: Sympathetic Nervous System; CDS: Coding sequence; GFP: Green fluorescent protein; oligos: Oligonucleotides; DMSO: Dimethyl Sulfoxide; DAPI: 4',6-diamidino-2-phenylindole; RIPA: Radio Immunoprecipitation Assay; PVDF: Poly vinylidene fluoride; TCGA: The cancer genome atlas; CGGA: Chinese Glioma Genome Atlas; WHO: World Health Organization; OS: Overall survival.
\end{abstract}

\section{Supplementary Information}

The online version contains supplementary material available at https://doi. org/10.1186/s12885-022-09330-9.

Additional file 1.

\section{Acknowledgements}

The authors would like to thank Cong Xue, Yu-Xin Chen, Xiao-Tian Wang, Feng Li, Bo-Min Guo and Ying-Rui Tian, in the Institute of Military Cognitive and Brain Science, Academy of Military Medical Sciences for their technical assistance.

\section{Authors' contributions}

LQ and XW designed the study. XW, ZS, YW, ZZ, FX, YZ, SW, YZ, HH contributed to acquiring the data. XW performed the statistical analysis and drafting of the manuscript. LQ and FX performed the revision of the manuscript. YW, FX and XW revised the manuscript. All authors read and approved the final manuscript.

\section{Funding}

This work was supported by grants from Beijing Natural Science Foundation (grant no. 5222033) and National Natural Science Foundation of China (grant nos. 81702454, 31771290).

\section{Availability of data and materials}

All data generated or analysed during this study are included in this published article.

\section{Declarations}

Ethics approval and consent to participate

The study was approved by the Academic Ethics and Security Committee of Academy of Military Medical Sciences (No. AF/SC-08/02.135).

\section{Consent for publication}

Not applicable.

\section{Competing interests}

The authors declare that they have no competing interests.

\section{Author details}

${ }^{1}$ Institute of Military Cognition and Brain Sciences, Academy of Military Medical Sciences, \#27 Taiping Road, Haidian, Beijing 100850, China. ${ }^{2}$ Laboratory Animal Center, North China University of Science and Technology, Tangshan 063210, Hebei, China. ${ }^{3}$ Qingdao Eighth People's Hospital, Qingdao 266041, Shandong Province, China.

Received: 24 June 2021 Accepted: 17 February 2022

Published online: 26 February 2022

\section{References}

1. Ostrom QT, Patil N, Cioffi G, et al. CBTRUS Statistical Report: Primary Brain and Other Central Nervous System Tumors Diagnosed in the United States in 2013-2017. Neuro Oncol. 2020;22:iv1-96.

2. Chinot OL, WickW, Mason W, et al. Bevacizumab plus radiotherapytemozolomide for newly diagnosed glioblastoma. N Engl J Med. 2014;370:709-22.

3. Louis DN, Perry A, Wesseling P, et al. The 2021 WHO classification of tumors of the central nervous system: a summary. Neuro-Oncology. 2021;23:1231-51.

4. Cloughesy TF, Cavenee WK, Mischel PS. Glioblastoma: from molecular pathology to targeted treatment. Annu Rev Pathol. 2014;9:1-25.

5. Naumann U, Harter PN, Rubel J, et al. Glioma cell migration and invasion as potential target for novel treatment strategies. Translat Neurosci. 2013:4:314-29.

6. Cuddapah VA, Robel S, Watkins S, et al. A neurocentric perspective on glioma invasion. Nat Rev Neurosci. 2014;15:455-65.

7. Antoni MH, Lutgendorf SK, Cole SW, et al. The influence of bio-behavioural factors on tumour biology: pathways and mechanisms. Nat Rev Cancer. 2006:6:240-8.

8. Cui B, Luo Y, Tian P, et al. Stress-induced epinephrine enhances lactate dehydrogenase $\mathrm{a}$ and promotes breast cancer stem-like cells. J Clin Invest. 2019;129:1030-46. 
9. Yang H, Xia L, Chen J, et al. Stress-glucocorticoid-TSC22D3 axis compromises therapy-induced antitumor immunity. Nat Med. 2019;25:1428-41.

10. Le CP, Nowell CJ, Kim-Fuchs C, et al. Chronic stress in mice remodels lymph vasculature to promote tumour cell dissemination. Nat Commun. 2016;7:10634

11. Krizanova O, Babula P, Pacak K. Stress, catecholaminergic system and cancer. Stress. 2016;19:419-28.

12. Thaker PH, Han LY, Kamat AA, et al. Chronic stress promotes tumor growth and angiogenesis in a mouse model of ovarian carcinoma. Nat Med. 2006;12:939-44.

13. Kim-Fuchs C, Le CP, Pimentel MA, et al. Chronic stress accelerates pancreatic cancer growth and invasion: a critical role for beta-adrenergic signaling in the pancreatic microenvironment. Brain Behav Immun. 2014;40:40-7.

14. Reiche EM, Nunes SO, Morimoto HK. Stress, depression, the immune system, and cancer. Lancet Oncol. 2004;5:617-25.

15. Zhi X, Li B, Li Z, et al. Adrenergic modulation of AMPKdependent autophagy by chronic stress enhances cell proliferation and survival in gastric cancer. Int J Oncol. 2019;54:1625-38.

16. Killock D. Skin cancer: propranolol limits melanoma recurrence. Nat Rev Clin Oncol. 2017;14:714

17. Daher $C$, Vimeux $L$, Stoeva $R$, et al. Blockade of beta-adrenergic receptors improves CD8(+) T-cell priming and Cancer vaccine efficacy. Cancer Immunol Res. 2019;7:1849-63.

18. Wang $X$, Sun W, Shen W, et al. Long non-coding RNA DILC regulates liver cancer stem cells via IL-6/STAT3 axis. J Hepatol. 2016;64:1283-94.

19. Zhang ZQ, Wang $X$, Xue $B H$, et al. Chronic stress promotes glioma cell proliferation via the PI3K/Akt signaling pathway. Oncol Rep. 2021;46.

20. He JJ, Zhang WH, Liu SL, et al. Activation of beta-adrenergic receptor promotes cellular proliferation in human glioblastoma. Oncol Lett. 2017;14:3846-52.

21. Mahabir R, Tanino M, Elmansuri A, et al. Sustained elevation of snail promotes glial-mesenchymal transition after irradiation in malignant glioma. Neuro-Oncology. 2014;16:671-85.

22. Zhou K, Zhang C, Yao H, et al. Knockdown of long non-coding RNA NEAT1 inhibits glioma cell migration and invasion via modulation of SOX2 targeted by miR-132. Mol Cancer. 2018;17:105.

23. Qu L, Ding J, Chen C, et al. Exosome-transmitted IncARSR promotes Sunitinib resistance in renal Cancer by acting as a competing endogenous RNA. Cancer Cell. 2016;29:653-68.

24. Yi L, Zhou X, LiT, et al. Notch1 signaling pathway promotes invasion, selfrenewal and growth of glioma initiating cells via modulating chemokine system CXCL12/CXCR4. J Exp Clin Cancer Res. 2019;38:339.

25. Xie F, Mei ZS, Wang X, et al. Loss of nuclear ARC contributes to the development of cardiac hypertrophy in rats. Acta Physiol (Oxf). 2020;228:e13337.

26. Gravendeel LA, Kouwenhoven MC, Gevaert O, et al. Intrinsic gene expression profiles of gliomas are a better predictor of survival than histology. Cancer Res. 2009;69:9065-72.

27. Wang Y, Qian T, You G, et al. Localizing seizure-susceptible brain regions associated with low-grade gliomas using voxel-based lesion-symptom mapping. Neuro-Oncology. 2015;17:282-8.

28. Liu X, Li Y, Qian Z, et al. A radiomic signature as a non-invasive predictor of progression-free survival in patients with lower-grade gliomas. Neuroimage Clin. 2018;20:1070-7.

29. Park SY, Kang JH, Jeong KJ, et al. Norepinephrine induces VEGF expression and angiogenesis by a hypoxia-inducible factor-1alpha protein-dependent mechanism. Int J Cancer. 2011;128:2306-16.

30. Batty GD, Russ TC, Stamatakis E, et al. Psychological distress in relation to site specific cancer mortality: pooling of unpublished data from 16 prospective cohort studies. BMJ. 2017;356:j108.

31. Yang EV, Kim SJ, Donovan EL, et al. Norepinephrine upregulates VEGF, IL-8, and IL-6 expression in human melanoma tumor cell lines: implications for stress-related enhancement of tumor progression. Brain Behav Immun. 2009;23:267-75.

32. Yang EV, Donovan EL, Benson DM, et al. VEGF is differentially regulated in multiple myeloma-derived cell lines by norepinephrine. Brain Behav Immun. 2008:22:318-23.

33. Bernabe DG, Tamae AC, Biasoli ER, et al. Stress hormones increase cell proliferation and regulates interleukin- 6 secretion in human oral squamous cell carcinoma cells. Brain Behav Immun. 2011:25:574-83.

34. Yang EV, Sood AK, Chen M, et al. Norepinephrine up-regulates the expression of vascular endothelial growth factor, matrix metalloproteinase
(MMP)-2, and MMP-9 in nasopharyngeal carcinoma tumor cells. Cancer Res. 2006;66:10357-64.

35. Sood AK, Bhatty R, Kamat AA, et al. Stress hormone-mediated invasion of ovarian cancer cells. Clin Cancer Res. 2006;12:369-75.

36. Quoc Lu'o'ng KV, Nguyen LT. The roles of beta-adrenergic receptors in tumorigenesis and the possible use of beta-adrenergic blockers for cancer treatment: possible genetic and cell-signaling mechanisms. Cancer Manag Res. 2012;4:431-45.

37. Barbieri A, Bimonte S, Palma G, et al. The stress hormone norepinephrine increases migration of prostate cancer cells in vitro and in vivo. Int J Oncol. 2015:47:527-34

38. Radu M, Semenova G, Kosoff R, et al. PAK signalling during the development and progression of cancer. Nat Rev Cancer. 2014;14:13-25.

39. Park MH, Lee HS, Lee CS, et al. p21-activated kinase 4 promotes prostate cancer progression through CREB. Oncogene. 2013;32:2475-82.

40. Cole SW, Sood AK. Molecular pathways: beta-adrenergic signaling in cancer. Clin Cancer Res. 2012;18:1201-6.

41. Udumyan R, Montgomery S, Fang F, et al. Beta-blocker drug use and survival among patients with pancreatic adenocarcinoma. Cancer Res. 2017;77:3700-7.

42. Al-Niaimi A, Dickson EL, Albertin C, et al. The impact of perioperative beta blocker use on patient outcomes after primary cytoreductive surgery in high-grade epithelial ovarian carcinoma. Gynecol Oncol. 2016;143:521-5.

43. Jansen $L$, Hoffmeister $M$, Arndt $V$, et al. Stage-specific associations between beta blocker use and prognosis after colorectal cancer. Cancer. 2014;120:1178-86.

44. Brabletz T, Kalluri R, Nieto MA, et al. EMT in cancer. Nat Rev Cancer. 2018;18:128-34.

45. Erin N, Grahovac J, Brozovic A, et al. Tumor microenvironment and epithelial mesenchymal transition as targets to overcome tumor multidrug resistance. Drug Resist Updat. 2020;53:100715.

46. Pastushenko I, Brisebarre A, Sifrim A, et al. Identification of the tumour transition states occurring during EMT. Nature. 2018;556:463-8.

47. Kahlert UD, Nikkhah G, Maciaczyk J. Epithelial-to-mesenchymal(-like) transition as a relevant molecular event in malignant gliomas. Cancer Lett. 2013;331:131-8.

48. Lu Y, Xiao L, Liu Y, et al. MIR517C inhibits autophagy and the epithelialto-mesenchymal (-like) transition phenotype in human glioblastoma through KPNA2-dependent disruption of TP53 nuclear translocation. Autophagy. 2015;11:2213-32.

49. Howe LR, Watanabe $\mathrm{O}$, Leonard J, et al. Twist is up-regulated in response to Wnt1 and inhibits mouse mammary cell differentiation. Cancer Res. 2003;63:1906-13.

50. Zhao Z, Rahman MA, Chen ZG, et al. Multiple biological functions of Twist1 in various cancers. Oncotarget. 2017;8:20380-93.

51. Cai $X$, Feng S, Zhang J, et al. USP18 deubiquitinates and stabilizes Twist 1 to promote epithelial-mesenchymal transition in glioblastoma cells. Am J Cancer Res. 2020;10:1156-69.

52. Cho KH, Jeong KJ, Shin SC, et al. STAT3 mediates TGF-beta1-induced TWIST1 expression and prostate cancer invasion. Cancer Lett. 2013:336:167-73.

53. Vichalkovski A, Gresko E, Hess D, et al. PKB/AKT phosphorylation of the transcription factor Twist-1 at Ser42 inhibits p53 activity in response to DNA damage. Oncogene. 2010;29:3554-65.

54. Li CW, Xia W, Huo L, et al. Epithelial-mesenchymal transition induced by TNF-alpha requires NF-kappaB-mediated transcriptional upregulation of Twist1. Cancer Res. 2012;72:1290-300.

55. Zheng Q, Diao S, Wang Q, et al. IL-17A promotes cell migration and invasion of glioblastoma cells via activation of PI3K/AKT signalling pathway. J Cell Mol Med. 2019;23:357-69.

56. Xiao T, Zhong W, Zhao J, et al. Polyphyllin I suppresses the formation of vasculogenic mimicry via Twist1 NE-cadherin pathway. Cell Death Dis. 2018;9:906.

57. Fu J, Chen Y, Cao J, et al. p28GANK overexpression accelerates hepatocellular carcinoma invasiveness and metastasis via phosphoinositol 3-kinase/AKT/hypoxia-inducible factor-1alpha pathways. Hepatology. 2011;53:181-92.

\section{Publisher's Note}

Springer Nature remains neutral with regard to jurisdictional claims in published maps and institutional affiliations. 